\title{
REAPRENDIZAJE MOTOR ORIENTADO A TAREAS EN PACIENTES CON SECUELAS DE ENFERMEDAD CEREBRO VASCULAR: UNA REVISIÓN NARRATIVA
}

Leidy Tatiana Ordoñez Mora', Tallia Katherine Araujo Morales², Lida Marlene Villacrez Pinchao ${ }^{3}$

\section{Resumen}

Introducción: en personas con secuelas de enfermedad cerebrovascular (ECV), la rehabilitación debe ser integral, con un enfoque funcional. En la actualidad, existe una técnica que lo posibilita desde un componente motriz, el reaprendizaje motor orientado a tareas (MRP). Como objetivo, se planteó establecer, a partir de la revisión de la literatura, los efectos de la técnica para miembros superiores, inferiores e independencia funcional en pacientes con secuelas de ECV. Métodos: se realizó una revisión narrativa a partir de consulta de bases de datos en línea (Pubmed, Elsevier, Lilacs, Scholar) y se incluyó literatura con información relevante acerca de fundamentación teórica. Resultados: el entrenamiento con esta técnica reporta efectos, en miembros inferiores, para mejoría de balance y marcha, y a nivel de miembros superiores, en funciones como alcances y agarres, lo que se traduce en una optimización de su independencia funcional. Conclusión: este programa puede ser una opción en la rehabilitación de la función motora en pacientes con estas secuelas.

Palabras clave: enfermedad cerebrovascular, hemiplejia, rehabilitación, fisioterapia (decs).

'Fisioterapeuta Especialista y Magíster en Neurorehabilitación, Docente Universidad Santiago de Cali, Contratista Secretaría de Deporte y Recreación, Cali. correo: tatiana.ormora@gmail.com

${ }^{2}$ Fisioterapeuta, Fundación SIRAD SAS. correo: tallia90@hotmail.com

${ }^{3}$ Fisioterapeuta, Fundación Universitaria María Cano, extensión Cali. correo: marlenevillacrez@gmail.com 


\section{TASK-ORIENTED MOTOR RELEARNING PROGRAM IN PATIENTS WITH SEQUELS OF VASCULAR BRAIN DISEASE: A NARRATIVE REVISION}

Leidy Tatiana Ordoñez Mora' ${ }^{1}$, Tallia Katherine Araujo Morales², Lida Marlene Villacrez Pinchao ${ }^{3}$

\section{Abstract}

Introduction: Rehabilitation must be comprehensive with a functional approach in people with sequels of Vascular Brain Disease VBD. There is a technique that allows this through a motor component named task-oriented motor relearning (TMR), it was established as objective to identify based on the literature review the effects of the technique for upper and lower limbs and functional independence in patients with sequels of VBD. Methods: A narrative review was carried out based on online databases (Pubmed, Elsevier, Lilacs, Scholar). Literature with relevant information about theoretical foundation was included. Results: The training with this technique reports effects on lower limbs to improve balance and gait, and for upper limbs in functions such as scopes and grips that results in an optimization of functional independence. Conclusion: This program may be an option for motor function rehabilitation in patients with these sequels. 


\section{REAPRENDIZAGEM MOTORA ORIENTADA POR TAREFA EM PACIENTES COM SEQUELAS DE DOENÇA CEREBROVASCULAR: UMA REVISÃO NARRATIVA}

Leidy Tatiana Ordoñez Mora', Tallia Katherine Araujo Morales², Lida Marlene Villacrez Pinchao ${ }^{3}$

\section{Resumo}

Introdução. A reabilitação deve ser parte integrante de uma abordagem funcional em pessoas com sequelas de doença cerebrovascular DCV. Existe uma técnica que o faz possível a partir de um componente motriz chamado reaprendizagem motora orientada por tarefa (MRP). O objetivo foi estabelecer a partir da revisão da literatura os efeitos da técnica para membros superiores e inferiores e independência funcional em pacientes com sequelas de DCV. Métodos. Foi realizada uma revisão narrativa a partir da busca em bases de dados online (Pubmed, Elsevier, Lilacs, Scholar), incluindo literatura com informações relevantes sobre fundamentação teórica. Resultados. $\mathrm{O}$ treinamento com essa técnica relata efeitos nos membros inferiores para melhorar o equilíbrio e a marcha, no nível dos membros superiores em funções como escopos e manoplas que resultam em uma otimização da independência funcional. Conclusão. Este programa pode ser uma opção na reabilitação da função motora em pacientes com essas sequelas 


\section{Introducción}

La enfermedad cerebrovascular (ECV) o accidente cerebrovascular (ACV) es un trastorno de la circulación cerebral, temporal o permanente, de una o varias áreas del encéfalo. Su etiología permite clasificarlo en: isquémico y hemorrágico(1,2,3). Después del primer año, más de la mitad de los casos presenta un déficit motriz, entre el 30 y $35 \%$ de los casos, en cognición y, en algunos casos, se acompaña de trastorno sensorial en lenguaje y deglución(4). En periodos inmediatos, se presenta espasticidad del hemicuerpo afectado y se incrementan los reflejos tónicos, así que se presenta resistencia a los estiramientos pasivos(5) y se compromete la calidad de las actividades de la vida diaria y actividades básicas cotidianas $(6,7)$. La ECV se presenta con mayor frecuencia en hombres, en población de raza negra, y puede ocurrir partir de los veinte años(8).

Su incidencia oscila en 700000 casos por año en los Estados Unidos, 35 a 183/100 000 habitantes/año para América Latina. La prevalencia es de un $80 \%$ de los pacientes que sobreviven a la etapa aguda y que, posteriormente, recuperan la capacidad de caminar. Por otro lado, entre un 30 a $66 \%$ de los pacientes sobrevivientes no pueden usar el miembro superior afectado, lo que conlleva deterioro funcional de la extremidad y afecta el desarrollo normal de sus actividades básicas cotidianas y de la vida diaria(9). En Latinoamérica, la prevalencia reportada oscila entre 1,74 a 6,51/1000. En Colombia, se presenta en 16/1000 habitantes en el departamento de Santander y en 1,42/1000 habitantes el departamento de Atlántico(10).
Los ECV generan alta mortalidad y las personas que sobreviven presentan, con frecuencia, deficiencia a nivel de estructuras corticales relacionadas con la planificación y ejecución del movimiento, limitaciones físicas, cognitivas, comunicativas o afectivas que impiden la ejecución de actividades de locomoción, autocuidado, entre otras, lo que se traduce en restricción de la participación y una subsecuente discapacidad funcional(11). La mala calidad de los movimientos y la dependencia funcional del individuo constituyen el principal objetivo terapéutico a intervenir(12). Por ello, los siguientes cuatro factores determinan la eficacia del tratamiento: tipo de rehabilitación hospitalaria, momento de inicio del tratamiento, intensidad y especificidad del tratamiento.

En muchos usuarios, la neurorehabilitación aparece como una opción de intervención que se ofrece desde el momento de ingreso al hospital y con continuidad de forma extrahospitalaria, esto se logra a partir del uso de estrategias cuyo objetivo es realizar movimientos útiles adaptados al medio que lo rodea $(13,14)$. Posteriormente, se trabaja con agarres y actividades finas de la mano $(15,16)$. Los objetivos fisioterapéuticos deben partir desde un modelo biopsicosocial $(17,18)$, $y$ deben centrarse en las consecuencias de la lesión, las características personales(19), y el entorno familiar y sociolaboral de la persona. Igualmente, se aplica de forma integral para lograr un alto nivel de independencia funcional y mejorar la calidad de vida cuando el paciente logra desenvolverse de forma independiente y se desempeña con un rol social en el domicilio y en la comu$\operatorname{nidad}(20,21,22)$. 
El reaprendizaje motor orientado a tareas o Motor Relearning Program (MRP) surge como una opción de rehabilitación a partir de investigación en neurociencia que incluye la práctica de tareas con la posibilidad de estimular el potencial de neuroplasticidad en el individuo(23) a partir del fraccionamiento $y$ direccionamiento en fases de la práctica de actividades; se emplean mecanismos de aprendizaje y de retroalimentación del movimiento dados por la repetición. Por lo tanto, el objetivo de este estudio es establecer, a partir de la revisión de la literatura, los efectos de la técnica para miembros superiores, inferiores e independencia funcional en pacientes con secuelas de ECV.

\section{Métodos}

Se realizó una revisión narrativa. Las estrategias para la identificación de los estudios incluyeron: búsqueda en las bases de datos en línea y bibliotecas virtuales con filtro por año para las publicaciones académicas entre 2000 y 2017 (Pubmed, Pedro, Elsevier, Google académico, Scielo, Ebsco, Proquest). Se usaron los siguientes términos de búsqueda: "motor relearning programme AND stroke", "motor relearning program AND stroke", "reaprendizaje motor orientado a tareas y accidente cerebro vascular", "reaprendizaje motor orientado a tareas y enfermedad cerebro vascular", "reaprendizaje motor orientado a tareas e ictus". Criterios de elegibilidad: los estudios debían ser ensayos clínicos, revisiones sistemáticas, estudios observacionales, estudios analíticos que incluyan los términos de búsqueda y con contenido acerca de los dos términos usados en el motor de búsqueda. Por el tipo de revisión, los artículos fueron incluidos a juicio de las autoras, para cada artículo se determinó una relevancia en la temática presentada, con el fin de documentar la técnica; no se tuvo en cuenta el idioma de publicación.

Se encontraron inicialmente 80 registros. Posteriormente, se descartaron registros duplicados y se obtuvieron los textos completos en donde las personas se sometían a una intervención. La información obtenida se consolidó en una matriz Excel, versión Windows 8, sobre la cual se consignó información detallada de los artículos encontrados con los criterios establecidos. Se incluyó el título del artículo, autor(es), país, año de publicación, tipo de artículo y temática central. Se recuperó la información tras la lectura analítica de los textos completos de los artículos para ofrecer sustento a la temática.

\section{Resultados}

La revisión de la literatura encontrada puede dividirse en los siguientes temas: efectos en miembros superiores e inferiores y efectos en independencia funcional (actividades de la vida diaria y estancia hospitalaria) en pacientes con secuelas de ECV.

El reaprendizaje motor orientado a tareas ha sido definido como una técnica de tratamiento que incluye la participación activa de los sujetos en un contexto seguro(24). Se basa en las teorías de sistemas(25), neurociencia y psicología cognitiva(26). El objetivo, básicamente, 
ECV para mejorar el control motor al realizar tareas o acciones esenciales. De este modo, se promueve el reaprendizaje a través de la enseñanza del movimiento(16). Es importante entender que este método se ha centrado en el paciente como aprendiz sumado a la necesidad de ejercicio, fuerza y entrenamiento, como medio para mejorar la capacidad, aprender las habilidades motoras y, así, optimizar el rendimiento motriz funcional(12).
El entrenamiento requiere acciones anticipatorias y práctica continua, y las tareas motoras involucradas se realizan dentro de un contexto que puede ser una tarea o entorno específico. Hay cuatro pasos descritos dentro del programa: análisis de la tarea, práctica del componente que falta, práctica de la tarea y transferencia de entrenamiento(27-28). La Tabla 1 ofrece una visión general de los procedimientos terapéuticos usados en cada uno de los estudios incluidos.

Tabla 1. Procedimientos terapéuticos en la rehabilitación de secuelas motoras de ECV. Criterio de inclusión universal: primer evento de ECV

\begin{tabular}{|c|c|c|c|c|c|c|c|c|}
\hline \multirow{3}{*}{ Autores } & \multirow{3}{*}{$\begin{array}{c}\text { No. De } \\
\text { pacientes }\end{array}$} & \multirow{3}{*}{$\begin{array}{l}\text { Fase del } \\
\text { ACV }\end{array}$} & \multirow{3}{*}{ Edad } & \multicolumn{4}{|c|}{ Grupos a intervenir } & \multirow{3}{*}{ Instrumentos de evaluación } \\
\hline & & & & \multicolumn{2}{|c|}{ Grupo 1} & \multicolumn{2}{|c|}{ Grupo 2} & \\
\hline & & & & No. pacientes & Técnica usada & No. pacientes & Técnica usada & \\
\hline $\begin{array}{l}\text { Langhammer y } \\
\text { Stangelle }\end{array}$ & 71 & Aguda & 49 - 95 años & 28 & Bobath & 33 & MRP & MAS, SMES, IB, NHP. \\
\hline Nilsson et al.:. & 73 & Aguda & $24-67$ años & 28 & $\begin{array}{l}\text { Caminar en } \\
\text { cinta rodante }\end{array}$ & 32 & MRP & $\begin{array}{c}\text { FIM, FAC, FMA, Escala de balance de } \\
\text { Berg y velocidad para caminar. }\end{array}$ \\
\hline $\begin{array}{l}\text { Langhammer y } \\
\text { Stangelle } e^{13}\end{array}$ & 61 & Aguda & 45 - 95 años & 28 & Bobath & 33 & MRP & MAS, SMES, IB, NHP. \\
\hline $\begin{array}{l}\text { Krutulyté et } \\
\text { al. }{ }^{32}\end{array}$ & 240 & Subaguda & $\begin{array}{l}\text { Promedio } \\
63 \text { años }\end{array}$ & 147 & Bobath & 93 & MRP & $\begin{array}{l}\text { IB; Escala de movilidad desarrollada } \\
\text { por la División de Investigación }\end{array}$ \\
\hline $\begin{array}{l}\text { Langhammer y } \\
\text { Stangelle }\end{array}$ & 16 & Crónica & $\begin{array}{l}\text { Promedio } \\
66 \text { años }\end{array}$ & 11 & MRP & 5 & Bobath & MAS, SMES, IB, NHP, Escala de Berg \\
\hline Chan et al..4 & 52 & Subaguda & $21-65$ años & 26 & MRP & 26 & $\begin{array}{l}\text { Terapia } \\
\text { convensional }\end{array}$ & $\begin{array}{l}\text { Escala de Berg, TUG, FIM, ADL, } \\
\text { Cuestionario de integración } \\
\text { comunitaria. }\end{array}$ \\
\hline $\begin{array}{l}\text { Langhammer y } \\
\text { Stangelle }{ }^{36}\end{array}$ & 61 & Aguda & 45 - 95 años & 33 & MRP & 28 & Bobath & MAS, SMES, IB, NHP. \\
\hline Pandian et al. ${ }^{21}$ & 30 & Crónica & $47-51$ años & 15 & Brunnstrom & 15 & MRP & FMA \\
\hline $\begin{array}{l}\text { El-Bahrawy y } \\
\text { Elwishy }\end{array}$ & 40 & Crónica & 40 - 60 años & 20 & Bobath & 20 & MRP & $\begin{array}{l}\text { Dinamómetro de mano, electro } \\
\text { goniómetro, Escala modificada de } \\
\text { Ashworth. }\end{array}$ \\
\hline Bhalerao et al.? & 32 & Aguda & $\begin{array}{l}\text { Promedio } 34 \\
\text { años }\end{array}$ & 17 & MRP & 15 & Bobath & DGI, FAC, IB. \\
\hline Zhu et al. ${ }^{38}$ & 188 & Subaguda & $45-80$ años & 98 & $\begin{array}{l}\text { Acupuntura + } \\
\text { fisioterapia }\end{array}$ & 90 & $\begin{array}{l}\text { MRP + } \\
\text { Brunstrom }\end{array}$ & FMA, IB. \\
\hline Rehami et al. ${ }^{28}$ & 12 & $\begin{array}{l}\text { Aguda y } \\
\text { Subaguda }\end{array}$ & $45-65$ años & 6 & MRP & 6 & $\begin{array}{l}\text { Terapia de } \\
\text { espejo }\end{array}$ & Escala de CAHAI, MMSE. \\
\hline $\begin{array}{l}\text { Bhalerao et } \\
\text { al. }^{39}\end{array}$ & 22 & Aguda & & 12 & MRP & 10 & Bobath & FAM, MAS, BI, FIM, FAC, DGI. \\
\hline Lord et al.: & 31 & Subaguda & $\begin{array}{l}\text { Promedio } 64 \\
\text { años }\end{array}$ & 16 & MRP & 14 & Comunidad & Marcha en 6 minutos, SIPSO, BBS. \\
\hline Xue et. $\mathrm{Al}^{41}$ & 150 & Aguda & $\begin{array}{l}\text { Promedio } \\
58-59\end{array}$ & 78 & MRP + Bobath & 72 & $\begin{array}{l}\text { Tratamiento } \\
\text { hospitalario }\end{array}$ & FMA, BI, NDS \\
\hline Plumer et, $\mathrm{Al}^{12}$ & 44 & Crónica & $\begin{array}{l}\text { Mayores de } \\
18 \text { años }\end{array}$ & 22 & MRP & 22 & $\begin{array}{l}\text { Entrenamiento } \\
\text { doble tarea } \\
\text { para marcha }\end{array}$ & FMA, TUG, ABCS \\
\hline
\end{tabular}


Se tomaron como protocolos de intervención las guías desarrolladas por Janet Carr y Roberta Shepherd en el 2003 para el tratamiento postictus, que tienen como objetivo presentar planes de intervención según la técnica y ejemplos en la práctica. Se basan en el desarrollo de tareas funcionales concretas(28), orientadas a la recuperación del balance corporal, la marcha, las transiciones de bípedo y sedente para miembros infe- riores, y la manipulación y alcance para miembros superiores. La intensidad de las intervenciones descritas en los artículos incluidos se menciona en la Tabla 2. Los estudios de Krutulytè(32) y Langhammer(33) no especifican la intensidad del entrenamiento que utilizaron en los grupos de intervención.

La Tabla 2 especifica la intensidad del entrenamiento que se usó en cada estudio.

Tabla 2. Intensidad del entrenamiento Fuente: Elaboración propia.

\begin{tabular}{|c|c|c|c|}
\hline Autores & Tiempo sesión & Días por semana & Tiempo entrenamiento \\
\hline Langhammer y Stanghelle $e^{29}$ & $40 \mathrm{~min}$ & 5 & Hasta el alta hospitalaria \\
\hline Nilsson et al. ${ }^{30}$ & $3 \min$ & 5 & 10 semanas \\
\hline Langhammer y Stanghelle ${ }^{35}$ & $40 \mathrm{~min}$ & 5 & 12 semanas \\
\hline chan et al. ${ }^{34}$ & 2 horas & No especifíca & 6 semanas \\
\hline Langhammer y Stanghelle $e^{35}$ & $40 \mathrm{~min}$ & 5 & 12 semanas \\
\hline Pandian et al. ${ }^{21}$ & 1 hora & 3 & 4 semanas \\
\hline El Bahrawy y El wishy ${ }^{36}$ & 2 horas & 3 & 6 semanas \\
\hline Bhalerao et al. ${ }^{37}$ & 1 hora & 6 & 6 semanas \\
\hline Zhu et al. ${ }^{38}$ & 4 horas & 5 & 12 semanas \\
\hline Rehani et al. ${ }^{28}$ & 1 hora & 6 & 4 semanas \\
\hline Bhalerao et al. ${ }^{39}$ & 1 hora & 6 & 6 semanas \\
\hline Lord et al. ${ }^{40}$ & 1 hora & 6 & 7 semanas \\
\hline Xue et al. ${ }^{41}$ & 3 veces al día durante 30 min & No especifíca & 1 mes \\
\hline Plummer et al. ${ }^{42}$ & $30 \min$ & 3 & 4 semanas \\
\hline
\end{tabular}

Fuente: Elaboración propia.

\section{Protocolos de intervención}

El entrenamiento del balance fue descrito en los estudios correspondientes a $(29,30,31,34,37)$, donde se incluían actividades de entrenamiento, actividad muscular y destreza. A medida que se adoptaban las tareas descritas en la posición sedente, se describían actividades en bípedo y las transferencias de peso con los componentes de entrenamiento y destreza ya mencionados. La transición de levantarse(32) se ejecutaba desde una posición inicial en sedente, con la parte superior del cuerpo vertical y los pies atrás, para que el paciente balanceara la parte superior del cuerpo por delante de las caderas y llegara a la bipedestación. Este ejercicio incluía secuencias de acción discriminadas, cambios en los tipos de sillas, posicionamiento del tronco, entre otros. 
Los protocolos de marcha $(29,30,31$, $34,37)$ se llevaban a cabo en situaciones de marcha con diferentes componentes musculares en superficies cambiantes, con el subsecuente entrenamiento de la destreza y los ejercicios funcionales con soporte de peso.

Las actividades de miembro superior para alcances y agarres $(21,27)$ variaban entre levantar un objeto con desviación radial de muñeca o con supinación de antebrazo, moverlo a la derecha y a la izquierda con flexoextensión de muñeca, sostener un vaso plástico lleno de agua sin deformarlo y pasarlo de una mano a otra y moverlo en diferentes direcciones, entre otros. En esta etapa también se incluía el manejo de la destreza y aumento en la dificultad y complejidad de los ejercicios.

Los estudios de Krutulyté (32), Langhammer (33), Langhammer (35), Zhu (38) y Bhalerao (39) no especifican los protocolos de intervención que utilizaron dentro de la práctica.

\section{Efectos en miembros superiores}

En cuanto a efectos del MRP en miembros superiores, se reporta que tras la aplicación del MRP mejora la calidad del movimiento y su número de repeticiones. Se pueden observar los resultados desde el tercer día(29-34). Otros ensayos comparan los resultados del MRP en cuanto a mejora de la función motora del miembro superior, con técnicas como Bobath y la terapia de espejo, las cuales no revelan diferencias significativas entre los grupos intervenidos, pero sí comprueban parcialmente que el
MRP ofrece mejores resultados en cuanto a tiempo de recuperación y calidad del movimiento(27-38).

Si bien el MRP obtuvo una mejora sustancial en cuanto a la recuperación de la fuerza de agarre de la mano y la disminución de la espasticidad en desviación ulnar, no se observó mejor en las funciones finas de la mano y la espasticidad flexora de la muñeca(36). Los resultados contrastan con otro estudio que tenía como objetivo efectuar una comparación de los protocolos de recuperación de la mano de MRP y la terapia de Brunstrom. Se encontró que este último recupera la función motora manual (masa flexo-extensora y de agarre) en menor tiempo, aunque no se encontraron diferencias significativas en cuanto al control de muñeca y coordinación del movimiento entre el MRP y Brunnstrom(21). Estudios aportaron que los resultados suelen mantenerse hasta los 6 meses, aproximadamente(27). A largo plazo, los efectos medidos en función motora, cantidad y calidad de movimiento tendían a disminuir rápidamente en los dos grupos intervenidos (MRP y Bobath)(37).

\section{Efectos en miembros inferiores}

En cuanto a efectos del MRP en miembros inferiores, fue posible demostrar que se puede lograr la deambulación funcional alrededor de la sexta semana, al igual que mejorar la velocidad de la marcha, pasando de $0,5 \mathrm{~m} / \mathrm{s}$ a $0,6 \mathrm{~m} / \mathrm{s}$ al alta médica. La marcha de manera independiente se consigue a la sexta semana, aproximadamente(37) y se mantiene hasta el sexto(43-40) y décimo mes(30). 
El equilibrio y el balance de las extremidades inferiores son las responsables de la deambulación funcional del paciente. El MRP mejora el equilibrio y disminuye el riesgo de caídas, dado que pasa de moderado a leve(31-30-34) y, en comparación con la terapia convencional, las personas que no lograron la deambulación de manera independiente se mantuvieron en silla de ruedas(29-31-32). La transición de bípedo a sedente se realiza con mayor facilidad en los grupos intervenidos con el MRP(35). No obstante, los resultados a largo plazo no se mantienen, ya que a los 4 años se evidencia una marcada pérdida del equilibrio con alto riesgo de caída(33).

\section{Independencia funcional}

La mayoría los artículos incluidos eran evaluados por el índice de Barthel. Los resultados obtenidos no mostraron diferencias significativas entre los grupos en comparación, excepto para las actividades de control de vejiga e intestino y para la independencia en situaciones de aseo personal, los cuales fueron visibles a los 3 meses(29-31-37-34), aunque, como se ha mencionado, los resultados no continúan a largo plazo(33). El MRP facilita la realización de actividades instrumentales de la vida diaria (AIVD) a partir de la segunda semana y se mantiene hasta la sexta semana tras la intervención(34).

\section{Discusión}

Los efectos en miembros superiores son evidenciados en periodos cortos de tiempo, desde la cuarta a la sexta semana, al igual que la mejora de la fuerza de agarre de la mano y la disminución de la espasticidad hacia la desviación ulnar de muñeca. No obstante, el enfoque Brunnstrom reporta mejores resultados en cuanto a la recuperación funcional de la mano(29-31-25-21-36), en contraste con lo encontrado en función motora, ya que el MRP permite realizar de manera rápida actividades motoras gruesas medidas con la escala de valoración motora (MAS) que mide cantidad y calidad del movimiento, después de la tercera y/o cuarta semana con un menor gasto energético(29-31-38-44-45).

En miembros inferiores, el MRP mejora la dinámica de la marcha a través de la disminución de la espasticidad, el fortalecimiento muscular y el mejoramiento del equilibrio. Gracias al balance desarrollado en las extremidades inferiores para realizar traslados de bípedo a sedente, es posible mejorar la capacidad de recorrer mayores distancias con mayor velocidad alrededor de la sexta semana, independientemente del empleo de ayudas externas(30-46-35-37-40).

Esto se puede atribuir al papel que desempeña el paciente en su proceso rehabilitatorio, puesto que, junto al terapeuta, determinan las actividades a entrenar según las necesidades del paciente en un contexto ambiental particular. Por esta razón, la retroalimentación juega un papel crucial en la rehabilitación, ya que proporciona un estímulo potencializador de la actividad. El avance en el reaprendizaje de las actividades induce grandes ajustes posturales de mantenimiento y anticipación del movimiento a ejecutar. En cuanto a la marcha, los efectos se pueden relacionar con las adaptaciones biomecánicas realizadas por el 
miembro inferior durante la ejecución, de modo que se asegura la extensión de la cadera en la fase de apoyo y el cumplimiento de la relación tiempo/apoyo de las extremidades (47-48).

El desempeño funcional incide directamente en las actividades de la vida diaria que, según los artículos revisados, fueron evaluadas con el índice de Barthel. Se presentaron mejores resultados en cuanto al control de vejiga $y$ función intestinal después de 2 a 3 meses, tomando como medida inicial la incapacidad del desplazarse de manera independiente. Las mediciones finales determinaron que la mayoría de los pacientes logran ir al baño de manera independiente, con supervisión, y un bajo porcentaje se mantiene en cama y en silla de ruedas. Por ende, es posible determinar que el MRP mejora significativamente la capacidad de auto cuidado(29-31-47-45-32-34-49-44-37).

No obstante, estas medidas pueden ser generales y no determinan aspectos de las actividades de la vida diaria relacionadas directamente con balance o función de miembros superiores.

Es importante establecer protocolos que permitan una recuperación precoz en un ámbito hospitalario en un programa de MRP en fases agudas tras el ECV. En los artículos revisados, se encontró que disminuye aproximadamente 13 días de la estancia hospitalaria, en comparación con otras técnicas como Bobath y la terapia convencional, así que se facilita la realización de las actividades de la vida diaria(29-31-37-50-51).
Esto puede deberse a mecanismos de neuroplasticidad inicial, y así, se puede tomar como un punto de partida para las intervenciones futuras ocasionadas, a su vez, por el estímulo en la respuesta de reorganización cortical en las áreas motoras. Por ello, se producen mejoras en la función nerviosa (patrones cerebrales de activación) tanto en experimentos efectuados en monos como en humanos(26-41). Por otro lado, los protocolos son intensivos, con una frecuencia diaria y una duración entre 30 minutos a 1 hora y, por ello, se dificulta la posibilidad de generar réplicas en la práctica hospitalaria o en consulta externa de rehabilitación.

Como continuidad en las estrategias de entrenamiento basados en teorías de control motor orientado a tareas, se encontró que cada vez son mayores los enfoques como, por ejemplo, la terapia robótica, la realidad virtual y las terapias restrictivas que involucran la participación de los individuos en ellas.

Es importante entender que el proceso de remisión a rehabilitación en sistemas de salud como el colombiano ocasiona que la mayoría de personas sean remitidas cuando hay un proceso de instauración de sinergias, razón por la cual se limitaría la aplicabilidad y eficacia del tratamiento(52). Como una alternativa de continuidad de entrenamiento con actividades orientadas a la tarea, se encuentra el task-oriented training. A pesar de que no se incluyó literatura acerca de esta alternativa en el procesamiento de información de esta revisión, se propone como un nuevo punto de partida para futuras revisiones en el tema. 


\section{Conclusión}

El reaprendizaje motor orientado a tareas descrito por las fisioterapeutas Janeth Carr y Roberta Sheperd(47) puede ser una opción interventiva a usarse en la actualidad en procesos neurorestaurativos en el ser humano que han sufrido una lesión neurológica como las enfermedades cerebrovasculares. A partir de lo analizado en la literatura, se concluye que se obtuvo efectos positivos a nivel de miembros superiores para alcances y agarres reflejados en la ejecución de estos con mayor destreza. En miembros inferiores hay un mejor desempeño en

\section{Referencias}

1.Martínez E, Murie M, Pagola I, Irimia P. Enfermedades cerebrovasculares. Medicine[Internet]. 2011 [Citado 2017 ago 10];10(72):4871-81. Disponible en: http:// www.elsevierinstituciones.com/ficheros/ pdf/62/62v10n72a13191296pdf001

2.Marrugat $\mathrm{J}$, et al. Estimación de la incidencia poblacional y la mortalidad de la enfermedad cerebrovascular establecida isquémica y hemorrágica en 2002. Rev Esp de Cardiol. 2007;60(6):573-80.

3.Reed K. Quick reference to Occupational Therapy [Internet]. Austin: PROED;2014. Parte III, Nervous System Disorders [citado 2015 sep 7]; p. 323-328. Disponible en: http://www.proedinc.com/ downloads/13826samplepgs.pdf

4.Fernández $\mathrm{O}$, et al. La calidad de vida del paciente con accidente cerebrovascular: una visión desde sus posibles factores determinantes. Rev Neurol. 2001;32(8):725-31. la ejecución del patrón de marcha y aumento en las estrategias de balance. Respecto a independencia funcional, no hay resultados específicos que se reporten posterior a esta ejecución; no obstante, se encontró que facilita el desempeño de actividades instrumentales. Finalmente, aunque se presentan resultados positivos, se requiere continuar con los procesos de investigación relacionados con esta técnica.

\section{Conflictos de intereses}

Las autoras declaran no tener ningún conflicto de interés.

5.Rodríguez L, Serra Y, Pérez S, Palermo R. La espasticidad como secuela de la enfermedad cerebrovascular. Rev Cubana Med. 2004;43(2-3):1.

6. Estévez A, Estévez A, de Lázaro J, Hardy Y. Grado de discapacidad en pacientes hemipléjicos del "Policlínico Docente Universitario del Cerro". Rev Cubana de Med Gen Integr. 2012;28(4):682-93.

7. Fernández E, Ruiz A, Sánchez G. Tratamiento de la extremidad superior en la hemiplejia desde terapia ocupacional. Revista TOG [Internet]. 2010 [Citado 2015 sep 12];7(11):1-24. Disponible en:

http://www.revistatog.com/num11/pdfs/ original1.pdf

8.Silva $\mathrm{F}$, et al. Enfermedad cerebrovascular en Colombia. Rev. Colomb. Cardiol. 2006;13(2):85-9.

9.Arlette D. Descripción de la terapia 
el contexto clínico. Rev Mex Neuroci. 2012;13(4):223-32.

10. Silva F, Quintero C, Zarruk J. Comportamiento epidemiológico de la enfermedad cerebrovascular en la población colombiana. En: Asociación Colombiana de Neurología. Guía Neurológica 8: Enfermedad Cerebrovascular [Internet]. Bogotá: ACN; p. 21-29. [Citado 2017 sep 15] Disponible en: http://www.acnweb.org/ guia/g8cap2.pdf

11. Avezum A, et al. Stroke in Latin America: Burden of Disease and Opportunities for Prevention. Glob Heart. 2015;10(4):323-31.

12. Carr J, Shepherd R. The changing face of neurological rehabilitation. Rev. bras. fisioter. 2006;10(2):147-56.

13. Odriozola $F$, et al. Pronóstico de las secuelas tras la lesión cerebral. Med Intensiva; 2009;33(4):171-81.

14. Flórez M. Intervenciones para mejorar la función motora en el paciente con ictus. Rehabilitación. 2000;34(6):423-36.

15. Terradillos E, et al. La clasificación internacional de funcionamiento (CIF) en la elaboración de un protocolo de evaluación del daño cerebral. Trauma Fund MAPFRE. 2012;23(2):123-9.

16. Seneviratne C. A comparison of two rehabilitation therapies in the elderly stroke population. Indian Journal of Gerontology. 2013;27(1):162-77.

17. Organización Panamericana de la Salud. Implementación del modelo biopsicosocial para la atención de personas con discapacidad a nivel nacional [Internet]. San Salvador: Instituto Salvadoreño de Rehabilitación de Inválidos; 2011. [citado 2017 oct 28]. Disponible en: http://www.paho.org/els/index. php?option $=$ com_docman\&task $=$ doc view\&gid $=870 \& \mid$ temid $=324$

18. Organización Mundial de la Salud. Informe mundial sobre la discapacidad
[Internet]. 2011. [Citado 2017 oct 28]. Disponible en: http://www.who.int/disabilities/ world_report/2011/es/

19. Borell F. El modelo biopsicosocial en evolución. Med Clin (Barc). 2002;119(5):175-9.

20. De la Fuente B, Quevedo E, Jiménez A, Zavala M. Funcionalidad para las actividades de la vida diaria en el adulto mayor de zonas rurales. Archivos en medicina familiar. 2010;12(1):1-4.

21. Pandian S, Narayan K, Rajkumar E. Comparison of Brunnstrom movement therapy and motor relearning program in rehabilitation of post-stroke hemiparetic hand: A randomized trial. Journal of Bodywork \& Movement Therapies. 2012;16: 330-7.

22. Varela L, Chavez J, Galvez M, Mendez F. Funcionalidad en el adulto mayor previa a su hospitalización a nivel nacional. Rev. Med. Hered. 2005;16(3):165-71.

23. Bayón M, et al. Eficacia de nuevas terapias en la neurorrehabilitación del miembro superior en el paciente con Ictus. Rehabilitación. 2013;48(4):232-40.

24. Bhalerao B, Parab D. Effect of additional use of shoe raise on unaffected side along with motor relearning programme (mrp) on ambulation in chronic hemiplegics: A Hypothesis. JMT. 2015;3(1):11-14.

25. Woldag $\mathrm{H}$, Hummelsheim $\mathrm{H}$. Evidence-based physiotherapeutic concepts for improving arm and hand function in stroke patients. J Neurol. 2002;249:51828. Disponible en: https://www.ncbi.nlm. nih.gov/pubmed/12021939

26. Yin $Y$, et al. How does the motor relearning program improve neurological function of brain ischemia monkeys? Neural Regen Res. 2013;8(16):1445-54.

27. Rehani P, Kumari R, Midha D. Effectiveness of motor relearning programme and mirror therapy on hand functions in patients with stroke-a randomized clinical 
trial. International Journal of Therapies and Rehabilitation Research. 2015;4(3): p. 20-4.

28. Cano de la Cuerda R, et al. Teorías y modelos de control y aprendizaje motor. Aplicaciones clínicas en neurorrehabilitación. Neurología. 2015;30(1):32-41.

29. Langhammer B, Stanghelle J. Bobath or Motor Relearning Programme? A comparison of two different approaches of physiotherapy in stroke rehabilitation: a randomized controlled study. Clinical Rehabilitation. 2000;14:361-9.

30. Nilsson $L$, et al. Walking training of patients with hemiparesis at an early stage after stroke: a comparison of walking training on a treadmill with body weight support and walking training on the ground. Clinical Rehabilitation. 2001;15:515-27.

31. Langhammer B, Stanghelle J. Fysioterapi etter hjerneslag - en randomisert kontrollert studie. Tidsskr Nor Lægeforen. 2001;121:2805-9.

32. Krutulytė $G$, Kimtys $A$, Kriščiūnas $A$. Kineziterapijos metodu Bobath ir judesių mokymo programos efektyvumas reabilituojant ligonius, sirgusius galvos smegenų insultu. Medicina. 2003;39(9):889-95.

33. Langhammer B, Stanghelle J. Bobath or Motor Relearning Programme? A follow-up one and four years post stroke. Clinical Rehabilitation. 2003;17:731-4.

34. Chan D, Chan C, Au D. Motor relearning programme for stroke patients: a randomized controlled trial. Clinical Rehabilitation. 2006;20:191 -200.

35. Langhammer B, Stanghelle J. Can Physiotherapy after Stroke Based on the Bobath Concept Result in Improved Quality of Movement Compared to the Motor Relearning Programme. Physiother Res Int. 2011;69-80.

36. El-Bahrawy M, El-Wishy A. Efficacy of motor relearning approach on hand func- tion in chronic stroke patients. A controlled randomized study. Italian Journal of Physiotherapy. 2012;2(4):121-7.

37. Bhalerao G, et al. Comparison of Motor Relearning Program versus Bobath approach at every two weeks interval for improving activities of daily living and ambulation in acute stroke rehabilitation. International Journal of Basic and Applied Medical Sciences. 2013;3(3):70-7.

38. Zhu Y, et al. Acupuncture in Subacute Stroke: No Benefits Detected. Physical Therapy. 2013;93(11):1447-55.

39. Bhalerao G, Kulkarni V, Kapoor D. Comparison of Two Physiotherapy Approaches in Acute Stroke Rehabilitation: Motor Relearning Program Versus Bobath Approach. Journal of Orthopaedics and Rehabilitation. 2011;1:79-88.

40. Lord S, et al. How feasible is the attainment of community ambulation after stroke? A pilot randomized controlled trial to evaluate community-based physiotherapy in subacute stroke. Clin Rehabil. 2008;22:215-25.

41. Xue J, Bai L, Guo Q, Yang C. Efficacy of early intervention of motor relearning program on post-stroke hemiplemia: $\mathrm{A}$ randomized controlled observation. Neural Regeneration Research. 2006;1(3):277-9.

42. Plummer $P$, Kyvelidou $A$, Sternad D, Najafi B. Training dual-task walking in community-dwelling adults within 1 year of stroke: a protocol for a single-blind randomized controlled trial. BMC Neurology. 2012;12(129):1-8.

43. Pohl M, et al. Repetitive locomotor training and physiotherapy improve walking and basic activities of daily living after stroke: a single-blind, randomized multicentre trial. Clinical Rehabilitation. 2007;21:17-27.

44. Han C, Wang Q, Ping-ping M, Zhu Qi. Effects of intensity of arm training on hemiplegic upper extremity motor re- 
covery in stroke patients: a randomized controlled trial. Clinical Rehabilitation. 2012;27(1):75-81.

45. Platz T. Evidenzbasierte armrehabilitation eine systematische literaturübersicht. Nervenarzt. 2003;74:841-9.

46. Peurala S, Pitkänen K, Sivenius J, Tarkka I. How much exercise does the enhanced gait-oriented physiotherapy provide for chronic stroke patients? J Neurol. 2004;251:449-53.

47. Carr J, Shepherd R. Stroke Rehabilitation. Guidelines for exercise and training to optimize motor skill. Edinburgh: Butterworth Heinemann; 2003.

48. Cano de la Cuerda R, Collado S. Neurorrehabilitación: métodos específicos de valoración y tratamiento. Madrid: Editorial Médica Panamericana; 2012
49. Dickson T. Motor relearning programme for stroke patients: a randomized controlled trial. Clinical Rehabilitation. 2006;20(11):1017-8.

50. Gustavsen M, Jansen R, Kjendhal A. Motor Relearning Program approach improves short-term motor outcomes and reduces hospital stay after stroke. Australian Journal of Physiotherapy. 2002;48-59.

51. Fernández E, et al. La neurorrehabilitación como alternativa esencial en el abordaje terapéutico de las ataxias cerebelosas. Revista Cubana de Salud Pública. 2013;39(3):489-500.

52. Ordoñez-Mora LT, et al. Terapia de restricción del lado sano como opción de manejo de personas con secuelas de enfermedad cerebrovascular. Arch Med (Manizales). 2017;17(1):173-84. 